\title{
Epidermal growth factor receptor mutation enhances expression of vascular endothelial growth factor in lung cancer
}

\author{
MING-SZU HUNG ${ }^{1-3}$, I-CHUAN CHEN ${ }^{4}$, PAUL-YANN LIN ${ }^{5}$, JR-HAU LUNG $^{1}$, YA-CHIN LI ${ }^{1}$, \\ YU-CHING LIN ${ }^{1-3}$, CHENG-TA YANG ${ }^{6,7}$ and YING-HUANG TSAI ${ }^{1,7}$ \\ ${ }^{1}$ Division of Thoracic Oncology, Department of Pulmonary and Critical Care Medicine, Chang Gung Memorial Hospital, \\ Puzih, Chiayi 61363; ${ }^{2}$ Department of Medicine, College of Medicine, Chang Gung University, Taoyuan 33302; \\ ${ }^{3}$ Department of Respiratory Care, Chang Gung University of Science and Technology, Chiayi Campus, Puzih, \\ Chiayi 61363; ${ }^{4}$ Department of Emergency Medicine, Chang Gung Memorial Hospital, Puzih, Chiayi 61363; \\ ${ }^{5}$ Department of Anatomic Pathology, Dalin Tzu Chi Hospital, Buddhist Tzu Chi Medical Foundation, Chiayi 62247; \\ ${ }^{6}$ Division of Pulmonary and Critical Care Medicine, Chang-Gung Memorial Hospital, Taoyuan 33305; \\ ${ }^{7}$ Department of Respiratory Care, College of Medicine, Chang Gung University, Taoyuan 33302, Taiwan, R.O.C.
}

Received May 13, 2015; Accepted September 27, 2016

DOI: $10.3892 / \mathrm{ol} .2016 .5287$

\begin{abstract}
Epidermal growth factor receptor (EGFR) activation has been demonstrated to have a critical role in tumor angiogenesis. In the present study, the correlation between EGFR mutations and vascular endothelial growth factor (VEGF) was investigated in lung cancer cell lines and non-small-cell lung cancer (NSCLC) tumor tissues. VEGF levels were significantly increased in culture medium of lung cancer cells and NSCLC tissues with EGFR mutations (H1650 vs. A549, $\mathrm{P}=0.0399 ; \mathrm{H} 1975$ vs. A549, $\mathrm{P}<0.0001)$. Stable lung cancer cell lines expressing mutant (exon 19 deletion, E746-A750; exon 21 missense mutation, L858R) and wild-type EGFR genes were established. Significantly increased expression of VEGF and stronger inhibitory effects of gefitinib to VEGF expression were observed in exon 19 deletion stable lung cancer cells (exon 19 deletion vs. wild-type EGFR, $\mathrm{P}=0.0005$ ). The results of the present study may provide an insight into the association of mutant EGFR and VEGF expression in lung cancer, and may assist with further development of targeted therapy for NSCLC in the future.
\end{abstract}

\section{Introduction}

The epidermal growth factor receptor (EGFR) is an attractive target for anticancer therapy, as EGFR signaling is a pathway

Correspondence to: DrMing-Szu Hung orProfessor Ying-Huang Tsai, Division of Thoracic Oncology, Department of Pulmonary and Critical Care Medicine, Chang Gung Memorial Hospital, 6 W. Sec. Jiapu Road, Puzih, Chiayi 61363, Taiwan, R.O.C.

E-mail: m12049@adm.cgmh.org.tw

E-mail: chestmed@cgmh.org.tw

Key words: lung cancer, erpidermal growth factor receptor, vascular endothelial growth factor, mutation that has a significant role in the growth, proliferation and survival of numerous solid tumors, including non-small cell lung cancer (NSCLC) (1). Gefitinib (Iressa ${ }^{\circledR}$, also known as ZD1839; AstraZeneca, London, UK), a synthetic anilinoquinazoline and adenosine triphosphate (ATP) mimetic, is the first commercially available EGFR tyrosine kinase inhibitor (EGFR-TKI). Administered orally, gefitinib competes with ATP for the tyrosine kinase binding site on the EGFR and the resulting inhibition of autophosphorylation blocks downstream signaling (2). Gefitinib has minimal adverse effects, but tumor responses are observed in only 10-19\% of patients with chemotherapy-refractory advanced NSCLC (3). However, a subgroup of patients with NSCLC possessing specific mutations in the tyrosine kinase domain of the EGFR gene, which correlates with favorable clinical responsiveness to gefitinib therapy, has been noted (3). All mutations appear to be limited to exons 18, 19, 20 and 21 of the EGFR gene (4). Missense mutations in exon 21 (L858R) and in-frame deletions within exon 19 (delE746-A750) have been shown to be the most frequent EGFR-TKI sensitive mutations $(80 \%)$ in NSCLC $(5,6)$.

EGFR activation has been shown to be associated with the stimulation of tumor angiogenesis, and angiogenesis is essential to growth, proliferation and metastasis of cancer cells (7-14). The EGFR ligands, EGF and transforming growth factor (TGF)- $\alpha$, demonstrated angiogenic properties. Expression of EGFR has been reported to be associated with the expression of angiogenic factors, such as TGF- $\alpha$ and VEGF in human cancers $(7,15)$. Activation of EGFR by EGF and TGF- $\alpha$ also upregulated VEGF expression in human cancer cell lines $(8,9)$. Gefitinib, which blocked the EGFR signaling pathway via inhibition of phosphorylated AKT, was reported to exert anti-angiogenic effects by blocking EGF induced upregulation of VEGF and interleukin (IL)-8 in human cancer cell lines (10). Treatment of several EGFR/TGF- $\alpha$-coexpressing tumor cell lines with gefitinib also resulted in growth inhibition that was accompanied by a decreased production of VEGF, basic EGF and TGF- $\alpha$ (11). 
The above data suggest that the EGFR signaling pathway modulates angiogenesis by way of upregulation of VEGF or other key angiogenic factors. VEGF is a key stimulator of angiogenesis, which induces proliferation, differentiation and migration of endothelial cells (12). VEGF also increases the vascular permeability and induces the production of proteases involved in the modification of the extracellular matrix (12). In NSCLC patients, high serum VEGF level is associated with increasing intratumoral angiogenesis and poor prognosis (13). As the mutations in EGFR may lead to increased growth factor signaling, the present study hypothesized that NSCLC with EGFR mutations may have more potential in induction of angiogenesis. However, the association of EGFR mutations and the activities of angiogenic factors in lung cancer have not been previously studied to the best of our knowledge.

In the present study, the association of VEGF expression with EGFR mutation was investigated in lung cancer cells and NSCLC tissues. Lung cancer cell lines stably transfected with wild-type and mutant EGFR genes were also established. VEGF expression and inhibitory effects of gefitinib to VEGF expression were also evaluated in these cells.

\section{Materials and methods}

Cell culture. The NSCLC cell lines A549 (ATCC CCL-185), H460 (ATCC HTB-177), H1650 (ATCC CRL-5883) and H1975 (ATCC CRL-5908) were purchased from American Type Culture Collection (Manassas, VA, USA). H1650 and H1975 cell lines have EGFR mutations (delE746-A750 for H1650; L858R and T790 M for H1975) (14). Human umbilical vein endothelial cells (HUVECs; H-UV001) were purchased from Bioresource Collection and Research Center (Hsinchu City, Taiwan). Cells were grown in complete growth medium [Dulbecco's modified Eagle's medium (Lonza, Basel, Switzerland) for A549 and H460 cells; RPMI-1640 media (Lonza) for H1650 and H1975 cells] supplemented with $10 \%$ fetal calf serum (Thermo Fisher Scientific, Inc., Waltham, MA, USA), 30 ng/ml EGF (Invitrogen; Thermo Fisher Scientific, Inc.), $10 \mathrm{U} / \mathrm{ml}$ penicillin and $10 \mu \mathrm{g} / \mathrm{ml}$ streptomycin at $37^{\circ} \mathrm{C}$ and $5 \% \mathrm{CO}_{2}$. Cells were incubated for $72 \mathrm{~h}$ and the supernatant of growth medium was collected for detection of VEGF levels. HUVECs were grown in 90\% Medium 199 (Lonza) with $25 \mathrm{U} / \mathrm{ml}$ heparin (Lonza) and $30 \mu \mathrm{g} / \mathrm{ml}$ endothelial cell growth supplement (Lonza), adjusted to contain $1.5 \mathrm{~g} / 1$ sodium bicarbonate, $10 \mathrm{U} / \mathrm{ml}$ penicillin and $10 \mu \mathrm{g} / \mathrm{ml}$ streptomycin, as well as $10 \%$ fetal calf serum. Gefitinib and G418 were purchased from Sigma Aldrich (EMD Millipore, Billerica, MA, USA).

Tissues. Thirty-two NSCLC tissue samples were obtained from NSCLC patients undergoing surgical resection of the primary tumor between July 2006 and May 2009, after approval from the Institutional Review Board at Chang Gung Memorial Hospital (IRB nos. 100-1405B and 103-6693C1) and patients' signed consent were obtained. All tissue samples were requested from the tissue bank of Chang Gung Memorial Hospital. Formalin-fixed, paraffin-embedded tissue samples were converted into tissue microarray (TMA) blocks using an AutoTiss 1000 arrayer (EverBio Technology, Inc., New Taipei City, Taiwan). The quality of the TMA slides was confirmed by the pathologist using hematoxylin- and eosin-stained slides.
DNA extraction and EGFR mutation analysis. DNA was extracted from formalin-fixed paraffin embedded tumors using the QIAamp DNA FFPE Tissue kit (Qiagen GmbH, Hilden, Germany). A high sensitivity OncoFOCUS ${ }^{\mathrm{TM}}$ Panel version 1.0 developed by Sequenom (San Diego, CA, USA) was used for EGFR mutation analysis with the mass-spectroscopy based MassArray device (16).

RNA extraction, complementary (c)DNA synthesis and reverse transcription-polymerase chain reaction ( $R T-P C R)$. RNA was extracted using the RNeasy Mini kit (Qiagen $\mathrm{GmbH}$ ) from cell pellets according to the manufacturers' protocol. Total RNA was then reverse transcribed to cDNA using the iScript ${ }^{\mathrm{TM}}$ cDNA Synthesis kit (Bio-Rad Laboratories, Inc., Hercules, CA, USA). A total of $2 \mu \mathrm{l}$ of reverse-transcribed cDNA was subjected to RT-PCR using $\mathrm{iQ}^{\mathrm{TM}} \mathrm{SYBR}^{\circledR}$ Green supermix at a total volume of $20 \mu \mathrm{l}$ and the Bio-Rad CFX96 ${ }^{\mathrm{TM}}$ quantitative PCR system (Bio-Rad Laboratories, Munich, Germany). The following primers were used for PCR: VEGF, 5'-TTCTGC TGTCTTGGGTGCATTGG-3' (sense) and 5'-ATCTCTCCT ATGTGCTGGCCTT-3' (antisense) (17); and $\beta$-actin, 5'-CCT GGACTTCGAGCAAGAGATG-3' (sense) and 5'-AGGAAG GAAGGCTGGAAGAGTG-3' (antisense). A typical protocol included a $95^{\circ} \mathrm{C}$ denaturation step for $3 \mathrm{~min}$ followed by 35 cycles with $95^{\circ} \mathrm{C}$ denaturation for $20 \mathrm{sec}, 60.3^{\circ} \mathrm{C}$ annealing and extension for $30 \mathrm{sec}$. Detection of the fluorescent product was performed at the extension step using a PCR machine. Melting curve detection and analysis were performed by an additional 80 cycles with a $55^{\circ} \mathrm{C}$ denaturation, with a $0.5^{\circ} \mathrm{C}$ increase following each cycle. Finally, the RT-PCR products were kept at $4^{\circ} \mathrm{C}$. Relative VEGF expression was analyzed by the $2^{-\Delta \Delta \mathrm{Cq}}$ method, using $\beta$-actin as the internal control (18).

Establishment of lung cancer stable cell lines expressing wild-type and mutant EGFR genes. Mutant EGFR genes (L858R and Del E746-A750) were generated by site-directed mutagenesis with specific primers from an expression vector harboring EGFR cDNA (pUSEAmpEGFRWt) (Upstate Biotechnology, Charlottesville, VA, USA). The following primers were used for site-directed mutagenesis: L858R, 5'-GATCACAGATTTTGGGCGGGCCAAACTGCTGGG-3' (sense) and 5'-CCCAGCAGTTTGGCCCGCCCAAAATCT GTGATC-3' (antisense); and Del E746-A750, 5'-CCCGTCGCT ATCAAAACATCTCCGAAAGCC-3' (sense) and 5'-GGC TTTCGGAGATGTTTTGATAGCGACGGG-3' (antisense). A QuikChange ${ }^{\mathrm{TM}}$ Site-Directed Mutagenesis kit (Stratagene California, San Diego, CA, USA) was used for site-directed mutagenesis according to the manufacturer's protocol. Cells were transfected with expression vectors using Lipofectamine ${ }^{\circledR} 2000$ reagent (Invitrogen; Thermo Fisher Scientific, Inc.) according to the manufacturer's protocol. A total of $48 \mathrm{~h}$ post transfection, cells were selected in G418 for 4 weeks, and cell colonies were selected and amplified for further studies.

Enzyme-linked immunosorbent assay (ELISA) of VEGF. The levels of VEGF in cell culture media were measured using a commercially available VEGF ELISA kit (Human VEGF ELISA kit; Biosource International, Inc., Camarillo, CA, USA) according to the manufacturer's protocol. The limits of sensitivity were $5 \mathrm{pg} / \mathrm{ml}$ for VEGF. 
Immunohistochemistry (IHC). Formalin-fixed, paraffin-embedded tissues were cut into $4-\mu \mathrm{m}$ sections, mounted on slides, deparaffinized with xylene and dehydrated using a gradient ethanol series. Stable lung cancer cells were cultured in a Nunc Lab-Tek ${ }^{\mathrm{TM}}$ II-Chamber Slide system (Thermo Fisher Scientific, Inc.), and subsequently fixed with formalin for further IHC study. Antigen retrieval was performed with citric acid $(\mathrm{pH}$ 6.0) at $97^{\circ} \mathrm{C}$ for $30 \mathrm{~min}$, followed by treatment with $3 \%$ hydrogen peroxide. The slides were incubated overnight at $4^{\circ} \mathrm{C}$ with antibodies against VEGF. The rabbit polyclonal VEGF antibody (catalog no., sc-152; Santa Cruz Biotechnology, Inc., Dallas, TX, USA) was used as primary antibody at a 1:50 dilution in TBS with $1 \%$ BSA. Following TBST washes, endogenous peroxidase activity was then quenched with $0.3 \%$ hydrogen peroxide in TBS. Mouse and rabbit specific HRP/DAB (ABC) detection IHC kit (catalog no., ab64264; Abcam, Cambridge, MA, USA) was then used according to the manufacturer's protocol. Detection was achieved using a biotinylated goat anti-rabbit secondary antibody (dilution, 1:1000; catalog no., ab6720; Abcam) and DAB chromogen. The sections were counterstained with hematoxylin before being mounted with organic media and glass slides. The IHC data for the specimens were assessed using the semi-quantitative immunoreactive score (IRS). The IRS was calculated by multiplying the staining intensity $(0=$ no staining, $1=$ weak staining, $2=$ moderate staining and $3=$ strong staining) by the percentage of positively stained cells $(0=0 \%$ of cells stained, $1=<10 \%$ of cells stained, $2=11-50 \%$ of cells stained, $3=51-80 \%$ of cells stained and $4=>81 \%$ of cells stained).

Protein extraction and western blot analysis. Whole cell proteins were extracted from lung cancer cells using M-PER Mammalian Protein Extraction Reagent (Pierce, Rockford, IL, USA) with Phosphatase Inhibitor Cocktail Set II (Calbiochem, San Diego, CA, USA) and Complete Protease Inhibitor Cocktail (Roche, Basel, Switzerland), according to the manufacturer's protocols. In total, $40 \mu \mathrm{g}$ protein were separated on $8 \%$ sodium dodecyl sulfate-polyacrylamide gels and transferred to Immobilon-P membranes (EMD Millipore). Membranes were incubated with primary antibodies against EGFR (catalog no., 2232; dilution, 1:500) phospho-EGFR (Tyr1068) (catalog no., 2220; dilution, 1:500) (both from Cell Signaling Technology, Inc., Danvers, MA, USA), $\beta$-actin (catalog no., A5441; dilution, 1:1,000) (Sigma-Aldrich; EMD Millipore), Akt (catalog no., sc-8312; dilution, 1:500), phospho-Akt (Ser473) (catalog no., sc-33437; dilution, 1:500), and $\gamma$-tubulin (catalog no., sc-12881; dilution, 1:1,000) (all from Santa Cruz Biotechnology, Inc., Dallas, TX, USA) at $4^{\circ} \mathrm{C}$ overnight. Subsequently, primary antibody and antigen complexes were bound to specific HRP-conjugated secondary antibodies [anti-rabbit (catalog no., sc-2340; dilution, 1:5,000), anti-goat (catalog no., sc-2953; 1:10,000), and anti-mouse (catalog no., sc-2371; dilution, 1:2,000) (all from Santa Cruz Biotechnology, Inc.)] at room temperature for $1 \mathrm{~h}$. An enhanced chemiluminescence blotting analysis system (GE Healthcare Life Sciences, Piscataway, NJ, USA) was used for antigen-antibody detection. The density of western blot bands was semi-quantified by ImageJ software (version 1.46; National Institutes of Health, Bethesda, MD, USA).

Transwell co-culture assay. HUVECs $\left(3 \times 10^{4}\right)$ were cultured in $35-\mathrm{mm} 6$ well dual-layered culture dishes at $37^{\circ} \mathrm{C}$. After $24 \mathrm{~h}$, wild-type and mutant EGFR transfected cells $\left(5 \times 10^{4}\right)$ were seeded onto the cell culture insert with $0.4-\mu \mathrm{m}$ micropores on the bottom (BD Biosciences, Franklin Lakes, NJ, USA) and placed in the wells growing HUVECs. HUVECs were collected on day 5 following co-culturing, and viable cells were then counted with a hemocytometer.

Statistical analysis. The Student's t-test was used to compare VEGF expression in various groups of samples. Statistical analysis was performed using SPSS (version 10.0; SPSS, Inc., Chicago, IL, USA). $\mathrm{P}<0.05$ was considered to indicate a significant difference, with two-sided analysis. All experiments were performed in triplicate.

\section{Results}

EGFR mutations and VEGF expression in lung cancer cells and NSCLC tissues. The association between EGFR mutations and VEGF expression was detected in lung cancer cell lines. VEGF levels in the culture media of lung cancer cell lines with mutant (H1975 and H1650) and wild-type EGFR (A549 and H460) were measured by ELISA (Fig. 1A). Significantly increased VEGF levels were noted in H1975 and H1650 lung cancer cell lines compared to the A549 cell line (Fig. 1A).

EGFR mutation statuses were analyzed in 32 NSCLC tissue samples. EGFR mutations were detected in 14 (41.6\%) samples, including 5 exon 21 L858R, 5 exon 19 deletions, 2 exon 21 L861Q and 1 exon 20 insertion mutation. Expression of VEGF was subsequently detected using IHC staining. Significantly increased expression of VEGF was noted in lung cancer tissues with EGFR mutations (Fig. 1B).

Establishment of lung cancer stable cells expressing wild-type and mutant EGFR genes. To further validate the association of mutant EGFR and expression of VEGF in an isogenic background, stable lung cancer cell lines expressing wild-type and mutant EGFR genes were subsequently established. A549 lung cancer cells were transfected with vectors containing wild-type and mutant EGFR genes. Following selection with G418, resistant cell colonies were selected and amplified. EGFR expression in cell colonies was detected by western blot analysis (Fig. 2A). Colonies with overexpression of EGFR were subsequently pooled and amplified for further analysis. Compared to wild-type EGFR gene transfected cells, increased expression of EGFR and phosphor-Akt proteins following EGF stimulation were detected in mutant EGFR gene transfected A549 lung cancer stable cells (Fig. 2B).

EGFR mutations and expression of VEGF in lung cancer stable cells. The expression of VEGF mRNA and protein were detected in mutant and wild type EGFR gene transfected stable lung cancer cells. Significantly increased expression of VEGF mRNA was noted in stable cells transfected with exon 19 deletion (E746-A750) mutant EGFR gene compared to those transfected with empty vector, wild-type and exon 21 missense (L858R) EGFR genes (Fig. 3A). Expression of VEGF protein was also observed in mutant and wild-type EGFR gene transfected stable lung cancer cells. Increased expression of VEGF protein was noted in stable cells transfected with exon 19 deletion (E746-A750) mutant 
A

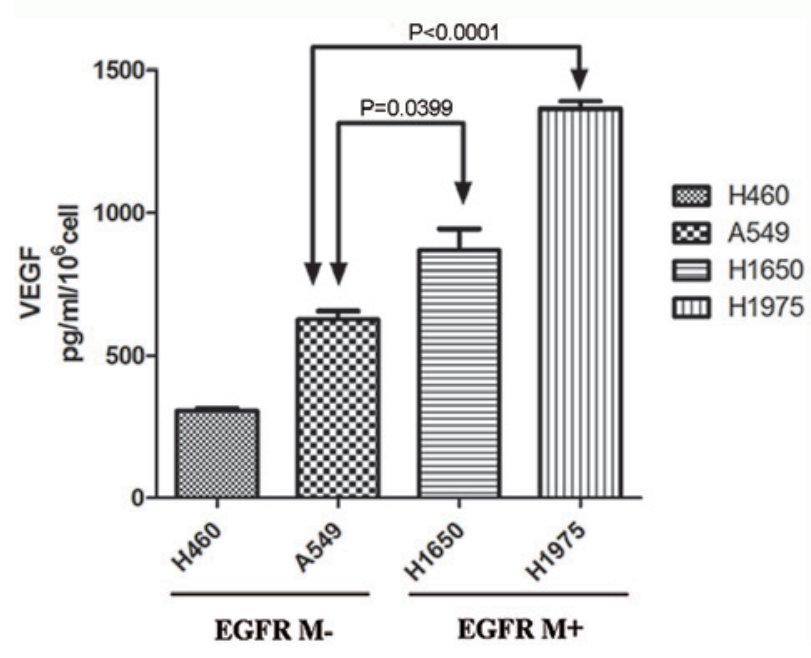

B

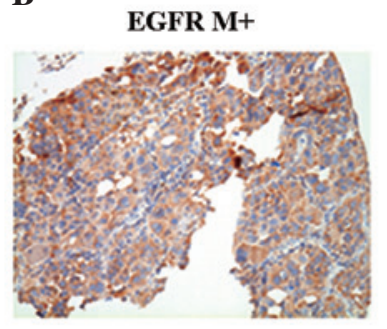

EGFR M-
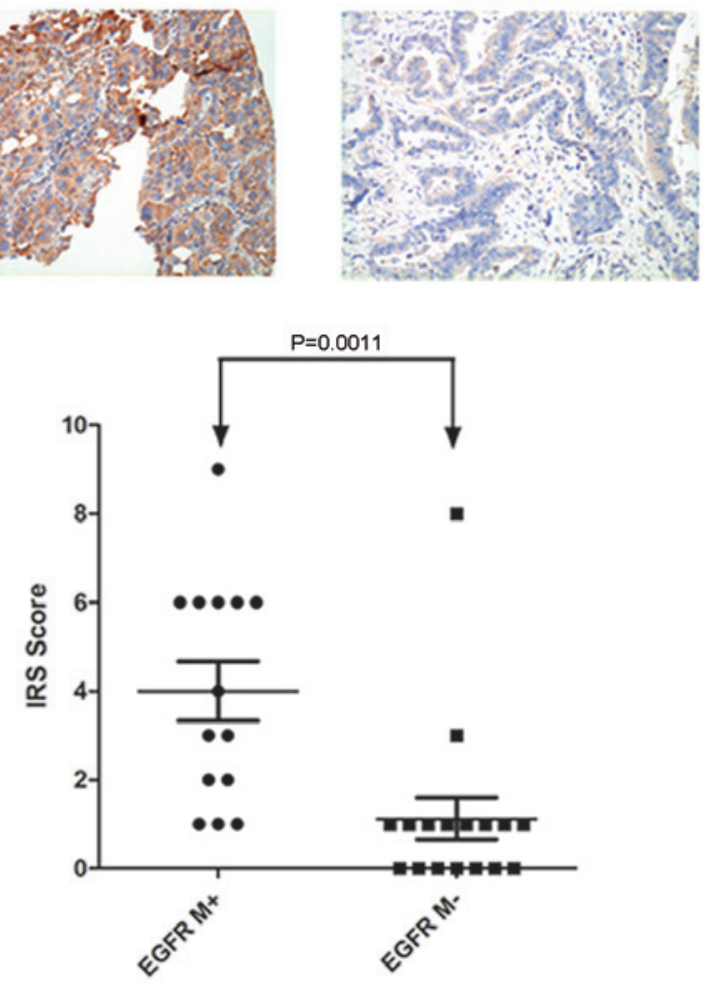

Figure 1. (A) VEGF levels secreted from cell lines with EGFR mutations (H1975 and H1650) and wild-type EGFR (A549 and H460). VEGF was measured by enzyme-linked immunosorbent assay as described previously. VEGF values are normalized to total cell numbers. Values are expressed as the means \pm SD of triplicate experiments. (B) VEGF expression was determined by immunohistochemical staining in mutant and wild-type non-small cell lung cancer tissues. The expression of VEGF was quantified by IRS and values are expressed as the mean \pm SD. Representative pictures for VEGF expression in EGFR mutant and wild-type non-small cell lung cancer tissues are also shown. EGFR M-, wild-type EGFR; EGFR M+, mutant EGFR. Magnification, x200. VEGF, vascular endothelial growth factor; EGFR, epidermal growth factor receptor; SD, standard deviation; URS, immunoreactive score.

A

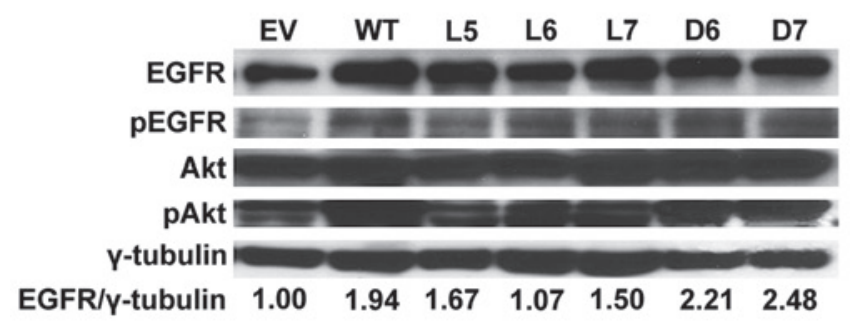

B

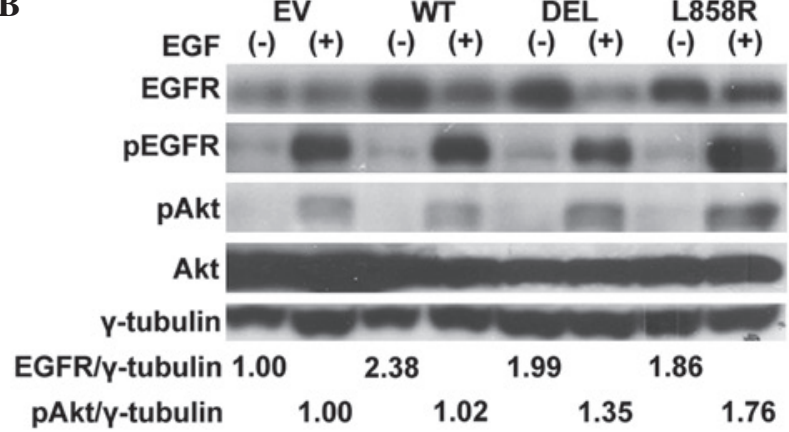

Figure 2. (A) Western blot analysis of EGFR and Akt expression/phosphorylation in EGFR transfected A549 stable cell lines. EGFR expression was normalized to the EV group using $\gamma$-tubulin as the internal control. (B) Western blot analysis of EGFR and Akt expression/phosphorylation in pooled EGFR transfected A549 stable cell lines with and without $100 \mathrm{ng} / \mathrm{ml}$ of EGF for $10 \mathrm{~min}$ following serum starvation for $48 \mathrm{~h}$. L5 and L7 clones were pooled as L858R. D6 and D7 clones were pooled as DEL. EGFR, epidermal growth factor receptor; EV, empty vector; WT, wild-type EGFR; L, L858R; D, DEL E746-A750; p, phophorylated.

EGFR gene by western blot (Fig. 3B) and IHC (Fig. 3C and D) analysis.

EGFR mutations and VEGF expression in lung cancer stable cells. Expression of VEGF in culture medium of EGFR transfected A549 stable lung cancer cells with/without EGF stimulation was measured. Significantly increased VEGF levels were noted in stable cells transfected with exon 19 deletion (Del E746-A750) mutant EGFR gene compared to those transfected with wild-type EGFR gene (Fig. 4A). A Transwell co-culture system was subsequently used to evaluate the ability of mutant EGFR genes to promote HUVEC growth. HUVECs were co-cultured with EGFR transfected A549 stable lung cancer cells in a Transwell system for 5 days. Significantly increased numbers of HUVECs were also noted in cells co-cultured with exon 21 missense (L858R) and exon 19 deletion (Del E746-A750) mutant EGFR genes (Fig. 4B).

EGFR mutations and angiogenesis in lung cancer stable cell lines. Inhibitory effects of gefitinib to VEGF in EGFR 
A
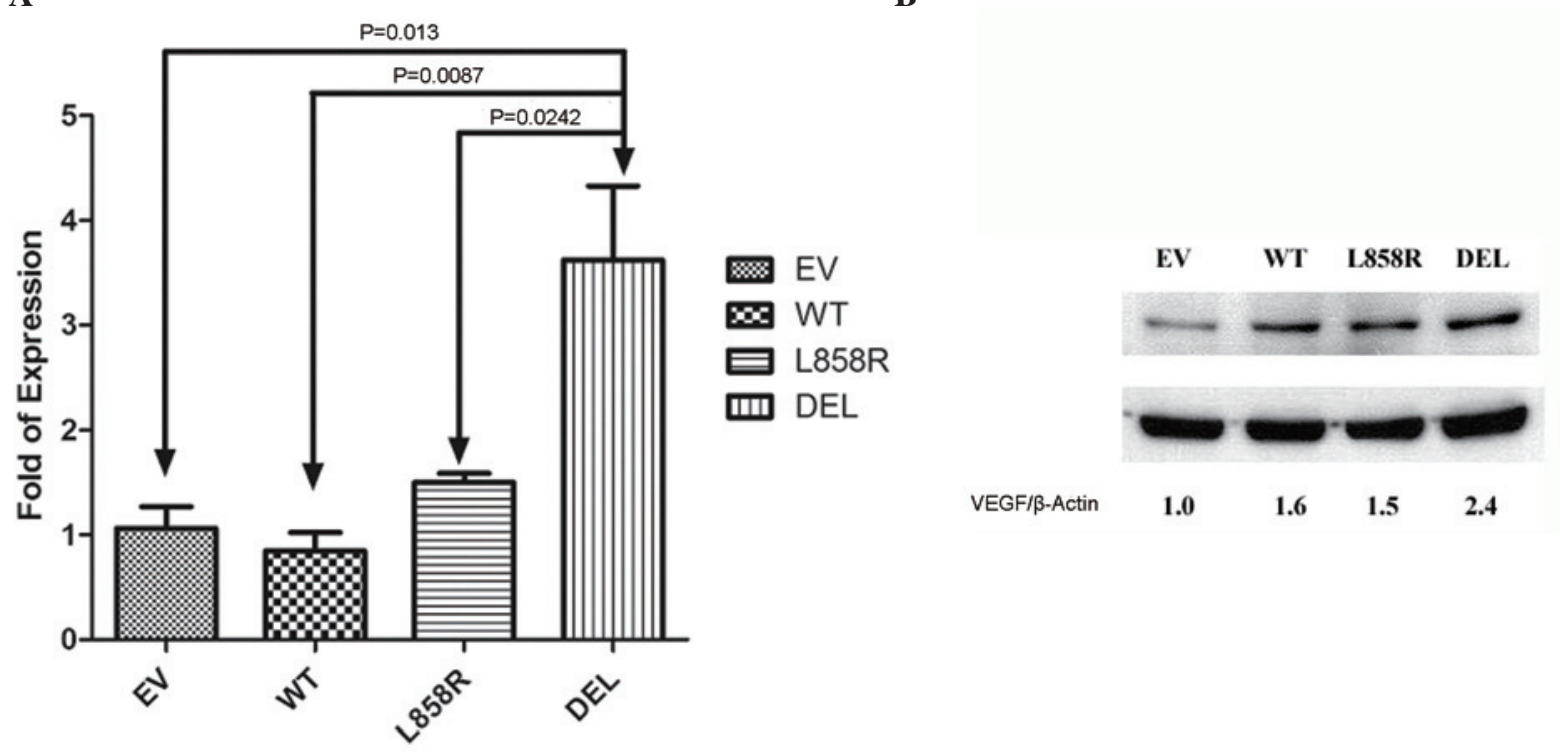

C

D

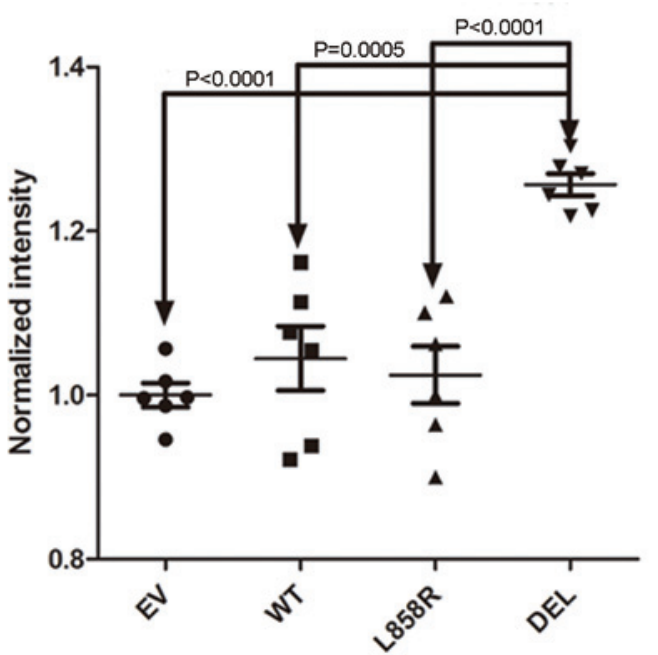

Figure 3. (A) Expression of VEGF mRNA in EGFR transfected A549 stable cells. (B) Western blot analysis of VEGF in EGFR transfected A549 stable cells. VEGF expression was normalized to the EV group using $\beta$-actin as the internal control. (C) IHC analysis of VEGF in EGFR transfected A549 stable cells. Magnification, $x 200$. (D) Intensities of IHC staining for VEGF were quantified. VEGF intensity values were normalized to the EV group. Values are expressed as the mean \pm standard deviation of six experiments. VEGF, vascular endothelial growth factor; EGFR, epidermal growth factor receptor; EV, empty vector; IHC, immunohistochemical; WT: wild-type EGFR.

transfected A549 stable lung cancer cells were also evaluated. Expression of VEGF in culture medium of EGFR transfected A549 stable lung cancer cells with EGF stimulation and gefitinib treatment was measured (Fig. 4C). Expression of VEGF following EGF stimulation was partially inhibited by gefitinib treatment. However, a significantly increased inhibition of VEGF was observed in stable cells transfected with exon 19 deletion (Del E746-A750) mutant EGFR gene (56\%) compared to those transfected with empty vector (33.6\%), wild-type EGFR (47.8\%) and exon 21 missense (L858R) mutation (37.1\%) genes (Fig. 4C).

\section{Discussion}

Association of EGFR mutations and VEGF expression has been reported in lung cancer tissues (19). In the present study, it was additionally observed that increased expression of VEGF is associated with EGFR mutations in lung cancer cells and NSCLC tissues. In order to elucidate these findings, stable lung cancer cell lines expressing exon 19 deletion (E746-A750), exon 21 missense (L858R) mutations and wild-type EGFR genes were established. Increased phosphorylation of EGFR and Akt were observed in stable lung cancer cell lines expressing exon 19 deletion (E746-A750) and exon 21 missense (L858R) mutations. It was observed that increased expression of VEGF is associated with overexpression of exon 19 deletion (E746-A750) EGFR gene and the expression of VEGF is inhibited by gefitinib, an EGFR-TKI. As a result, EGFR mutation may enhance VEGF expression through increased phosphorylation of EGFR and Akt in lung cancer cells. Increased proliferation rate was also observed in HUVECs co-cultured with exon 19 deletion and (E746-A750) exon 21 missense (L858R) mutation stable lung cancer cells. Furthermore, it was observed that the expression of VEGF in stable lung cancer cells is inhibited by gefitinib, which demonstrated increased inhibitory effects to expression of VEGF 
A

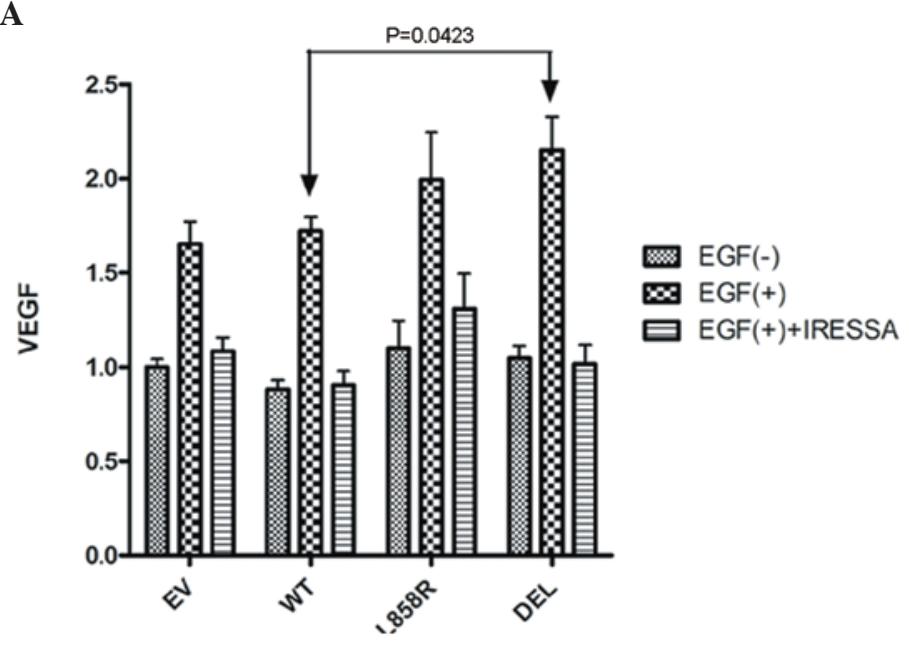

B

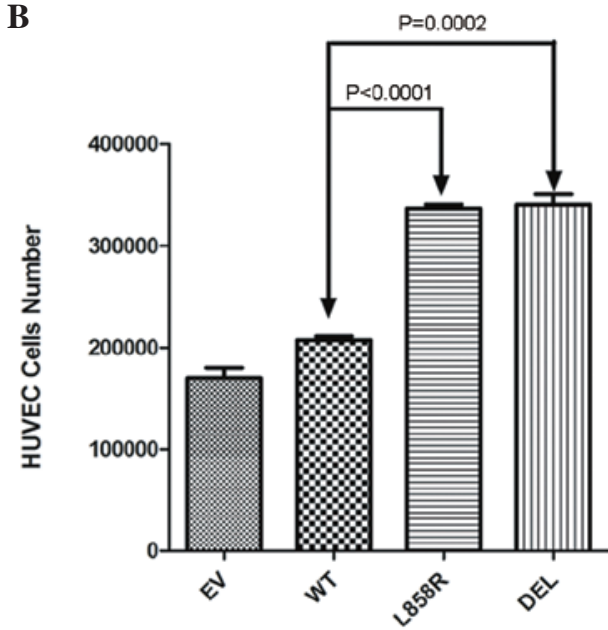

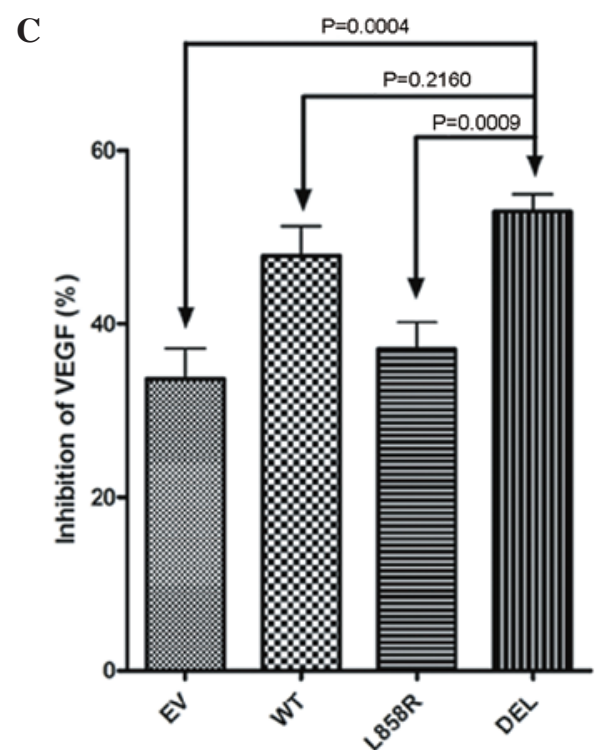

Figure 4. (A) Expression of VEGF in culture medium of EGFR transfected A549 stable lung cancer cell lines with or without EGF (100 ng/ml) stimulation for $24 \mathrm{~h}$ was measured by enzyme-linked immunosorbent assay. Groups with gefitinib $(20 \mu \mathrm{M})$ and EGF stimulation were also measured. Values are expressed as the mean $\pm \mathrm{SD}$ of triplicate experiments normalized to cell numbers and subsequently normalized to the group of empty vector transfected cells without EGF stimulation. (B) HUVECs were co-cultured with EGFR transfected A549 stable lung cancer cell lines in a Transwell system for 5 days. Cells in each group were counted. Values are expressed as the mean $\pm \mathrm{SD}$ of triplicate experiments. (C) Inhibition effects of VEGF in culture medium with EGF (100 ng/ml) stimulation for $24 \mathrm{~h}$ were measured following gefitinib treatment. Values are expressed as the mean $\pm \mathrm{SD}$ of triplicate experiments normalized to each group of EGFR transfected A549 stable lung cancer cell lines without gefitinib treatment. EV, Empty vector; WT, wild-type EGFR; VEGF, vascular endothelial growth factor; EGFR, epidermal growth factor receptor; SD, standard deviation; HUVECs, human umbilical vein endothelial cells; IRESSA, gefitinib.

in stable lung cancer cell lines expressing exon 19 deletion (E746-A750) mutation. EGFR-TKIs have been proposed to inhibit lung cancer cells through oncogenic shock by inhibiting phosphorylation of EGFR tyrokinase domain and downstream Akt signaling pathways (20). The results of the present study demonstrated that in addition to oncogenic shock, EGFR-TKIs may exert their effects through inhibition of VEGF expression and angiogenesis in lung cancer cells.

Both exon 19 deletion and exon 21 missense mutations are common EGFR mutations, and have been proven to be associated with a favorable response to gefitinib as well as other EGFR-TKIs, including erlotinib and afatinib (21-23). However, emerging evidence has suggested that exon 19 deletion mutation is associated with an improved outcome following afatinib therapy compared with exon 21 L858R mutation, which may suggest that these mutations have distinct features (22). In the present study, it was observed that exon 19 deletion mutation is associated with increased expression of VEGF. The expression of VEGF was inhibited by gefitinib in all lung cancer cells investigated. Notably, an increased inhibitory effect of gefitinib to the secretion of VEGF was observed in exon 19 deletion stable cells. As a result, the present study supports the finding that exon 19 deletion and exon 21 L858R mutation have distinct features. The results of the present study demonstrated that lung cancer cells harboring exon 19 deletion EGFR mutation may exert increased expression of VEGF and angiogenesis ability. In addition, gefitinib, as well as other EGFR-TKIs, may have increased inhibitory effects on these cells.

In the present study, both exon 19 deletion and exon 21 L858R mutation cells were observed to promote proliferation of HUVECs. As a result, other angiogenic factors in addition to VEGF may be associated with EGFR mutations. For example, mutant EGFR was reported to activate the gp130/JAK/STAT3 signaling pathway by means of IL-6 upregulation in primary 
human lung adenocarcinomas (24). In pancreatic cancer cells, inhibition of EGFR activation resulted in decreased expression of IL-8 (25). Taken together, mutant EGFR may contribute to the production of VEGF, IL-8, IL-6 and other angiogenic factors in lung cancer cells. The expression of VEGF was only partially inhibited by gefitinib in the present study, which implies that a combination of EGFR-TKI with anti-VEGF therapy may provide an improved response compared with EGFR-TKI treatment alone in lung cancer cells. This hypothesis is supported by a recent meta-analysis study, which demonstrated that the combination of erlotinib and bevacizumab, an anti-VEGF antibody, has an improved response compared with erlotinib treatment alone in advanced NSCLC (26). Further studies to investigate this hypothesis are ongoing in our laboratory.

In summary, the present study demonstrated that mutant EGFR is associated with increased expression of VEGF in NSCLC cells and tissues. Exon 19 deletion mutation stable cells were observed to have increased expression of VEGF and to be more susceptible to EGFR-TKI inhibition of VEGF expression. The results of the present study may provide an insight into the association between mutant EGFR and VEGF expression in lung cancer, and lead to further development of combined anti-EGFR and anti-VEGF therapy for NSCLC in the future.

\section{Acknowledgements}

The present study was supported by grant nos., CMRPG650181 (to MSH and CTY), CMRPG6B0231 (to MSH), CMRPG6B0232 (to MSH), CORPG6B0353 (to YHT and MSH) and CORPG6B0363 (to YCL and MSH) in Chang Gung Memorial Hospital (Chiayi, Taiwan). In addition, the authors would like to acknowledge the tissue microarray service provided by the Expensive Advanced Instrument Core Laboratory, Department of Medical Research and Development, Chang Gung Memorial Hospital.

\section{References}

1. Arteaga CL: Epidermal growth factor receptor dependence in human tumors: More than just expression? Oncologist 4 (Supp 7): S31-S39, 2002.

2. Paez JG, Jänne PA, Lee JC, Tracy S, Greulich H, Gabriel S, Herman P, Kaye FJ, Lindeman N, Boggon TJ, et al: EGFR mutations in lung cancer: Correlation with clinical response to gefitinib therapy. Science 304: 1497-1500, 2004

3. Maemondo M, Inoue A, Kobayashi K, et al: Gefitinib or chemotherapy for non-small-cell lung cancer with mutated EGFR. N Engl J Med 362: 2380-2388, 2010.

4. Gazdar AF, Shigematsu H, Herz J and Minna JD: Mutations and addiction to EGFR: The Achilles 'heal' of lung cancers? Trends Mol Med 10: 481-486, 2004.

5. Hsieh RK, Lim KH, Kuo HT, Tzen CY and Huang MJ: Female sex and bronchioloalveolar pathologic subtype predict EGFR mutations in non-small cell lung cancer. Chest 128: 317-321, 2005.

6. Shih JY, Gow CH, Yu CJ, et al: Epidermal growth factor receptor mutations in needle biopsy/aspiration samples predict response to gefitinib therapy and survival of patients with advanced nonsmall cell lung cancer. Int J Cancer 118: 963-969, 2006.

7. Bancroft CC, Chen Z, Yeh J, Sunwoo JB, Yeh NT, Jackson S, Jackson $C$ and Van Waes C: Effects of pharmacologic antagonists of epidermal growth factor receptor, PI3K and MEK signal kinases on NF-kappaB and AP-1 activation and IL-8 and VEGF expression in human head and neck squamous cell carcinoma lines. Int J Cancer 99: 538-548, 2002.

8. Goldman CK, Kim J, Wong WL, King V, Brock T and Gillespie GY: Epidermal growth factor stimulates vascular endothelial growth factor production by human malignant glioma cells: A model of glioblastoma multiforme pathophysiology. Mol Biol Cell 4: 121-133, 1993.
9. Ravindranath N, Wion D, Brachet P and Djakiew D: Epidermal growth factor modulates the expression of vascular endothelial growth factor in the human prostate. J Androl 22: 432-443, 2001.

10. Hirata A, Ogawa S, Kometani T, Kuwano T, Naito S, Kuwano M and Ono M: ZD1839 (Iressa) induces antiangiogenic effects through inhibition of epidermal growth factor receptor tyrosine kinase. Cancer Res 62: 2554-2560, 2002.

11. Ciardiello F, Caputo R, Bianco R, Damiano V, Fontanini G, Cuccato S, De Placido S, Bianco AR and Tortora G: Inhibition of growth factor production and angiogenesis in human cancer cells by $\mathrm{Z}$ (Iressa), a selective epidermal growth factor receptor tyrosine kinase inhibitor. Clin Cancer Res 7: 1459-1465, 2001.

12. Ferrara N: Vascular endothelial growth factor: Basic science and clinical progress. Endocr Rev 25: 581-611, 2004.

13. Shimanuki Y, Takahashi K, Cui R, Hori S, Takahashi F, Miyamoto $\mathrm{H}$ and Fukurchi Y: Role of serum vascular endothelial growth factor in the prediction of angiogenesis and prognosis for non-small cell lung cancer. Lung 183: 29-42, 2005.

14. Pao W, Miller VA, Politi KA, Riely GJ, Somwar R, Zakowski MF, Kris MG and Varmus H: Acquired resistance of lung adenocarcinomas to gefitinib or erlotinib is associated with a second mutation in the EGFR kinase domain. PLoS Med 2: e73, 2005.

15. Maeshima Y, Sudhakar A, Lively JC, Ueki K, Kharbanda S, Kahn CR, Sonenberg N, Hynes RO and Kalluri R: Tumstatin, an endothelial cell-specific inhibitor of protein synthesis. Science 295: 140-143, 2002.

16. Cote ML, Haddad R, Edwards DJ, Atikukke G, Gadgeel S, Soubani AO, Lonardo F, Bepler G, Schwartz AG and Ethier SP: Frequency and type of epidermal growth factor receptor mutations in African Americans with non-small cell lung cancer. J Thorac Oncol 6: 627-630, 2011.

17. Jia SF, Guan H, Duan X and Kleinerman ES: VEGF165 is necessary to the metastatic potential of Fas (-) osteosarcoma cells but will not rescue the Fas (+) cells. J Exp Ther Oncol 7: 89-97, 2008.

18. Livak KJ and Schmittgen TD: Analysis of relative gene expression data using real-time quantitative PCR and the 2(-Delta Delta C(T)) Method. Methods 25: 402-408, 2001.

19. Reinmuth N, Jauch A, Xu EC, Muley T, Granzow M, Hoffmann H, Dienemann H, Herpel E, Schnabel PA, Herth FJ, et al: Correlation of EGFR mutations with chromosomal alterations and expression of EGFR, ErbB3 and VEGF in tumor samples of lung adenocarcinoma patients. Lung cancer 62: 193-201, 2008.

20. Scaltriti M and Baselga J: The epidermal growth factor receptor pathway: A model for targeted therapy. Clin Cancer Res 12: 5268-5272, 2006.

21. Rosell R, Ichinose Y, Taron M, Sarries C, Queralt C, Mendez P, Sanchez JM, Nishiyama K, Moran T, Cirauqui B, et al: Mutations in the tyrosine kinase domain of the EGFR gene associated with gefitinib response in non-small-cell lung cancer. Lung Cancer 50: 25-33, 2005.

22. Yang JC, Wu YL, Schuler M, Sebastian M, Popat S, Yamamoto N, Zhou C, Hu CP, O'Byrne K, Feng J, et al: Afatinib versus cisplatin-based chemotherapy for EGFR mutation-positive lung adenocarcinoma (LUX-Lung 3 and LUX-Lung 6): Analysis of overall survival data from two randomised, phase 3 trials. Lancet Oncol 16: 141-151, 2015.

23. Zhou C, Wu YL, Chen G, Feng J, Liu XQ, Wang C, Zhang S, Wang J, Zhou S, Ren S, et al: Erlotinib versus chemotherapy as first-line treatment for patients with advanced EGFR mutation-positive non-small-cell lung cancer (OPTIMAL, CTONG-0802): A multicentre, open-label, randomised, phase 3 study. Lancet Oncol 12: 735-742, 2011.

24. Gao SP, Mark KG, Leslie K, Pao W, Motoi N, Gerald WL, Travis WD, Bornmann W, Veach D, Clarkson B and Bromberg JF: Mutations in the EGFR kinase domain mediate STAT3 activation via IL-6 production in human lung adenocarcinomas. J Clin Invest 117: 3846-3856, 2007.

25. Bruns CJ, Solorzano CC, Harbison MT, Ozawa S, Tsan R, Fan D, Abbruzzese J, Traxler P, Buchdunger E, Radinsky R and Fidler IJ: Blockade of the epidermal growth factor receptor signaling by a novel tyrosine kinase inhibitor leads to apoptosis of endothelial cells and therapy of human pancreatic carcinoma. Cancer Res 60: 2926-2935, 2000.

26. Sun L, Ma JT, Zhang SL, Zou HW and Han CB: Efficacy and safety of chemotherapy or tyrosine kinase inhibitors combined with bevacizumab versus chemotherapy or tyrosine kinase inhibitors alone in the treatment of non-small cell lung cancer: A systematic review and meta-analysis. Med Oncol 32: 473, 2015. 